Check for updates

Cite this: RSC Adv., 2019, 9, 9301

\title{
Yellow fluorescent graphene quantum dots as a phosphor for white tunable light-emitting diodes $\uparrow$
}

\author{
Luqiao Yin, ${ }^{\text {ac }}$ Jipeng Zhou, ${ }^{\text {ad }}$ Weitao Li, ${ }^{\mathrm{b}}$ Jianhua Zhang ${ }^{\star a c}$ and Liang Wang (D) *b
}

In recent decades, quantum dots have been considered to be highly promising photoluminescent materials for white light devices. During the application of quantum dots in the fabrication of white LEDs, the spectrum and color temperature of the devices are modulated; these devices often involve quantum dots with different emission wavelengths. In this study, yellow-green emitting graphene quantum dots (GQDs) were fabricated using a simple, low-cost and eco-friendly method. The obtained GQDs were cast in UV-curable siloxane. Then, a polymer film with superior optical transparency and excellent monodisperse properties of GQDs was formed. Via the simple adjustment of thickness and the GQD concentration of the color convert matrix, tunable color temperatures (3196-10 $870 \mathrm{~K}$ ) of the GQDbased white light-emitting diodes (LEDs) were achieved. The CIE (Commission Internationale de L'Eclairage) coordinates of the GQD-based white light-emitting diodes matched well with the blackbody radiation curve. Using the fluorescent polymeric matrix in white LEDs, good quality emission and gratifying stability could be obtained. Moreover, this indicates that this technology has the potential for applications in high-end lighting.

Received 17th December 2018

Accepted 7th March 2019

DOI: 10.1039/c8ra10353d

rsc.li/rsc-advances a wavelength conversion layer, and the blue-chip serves as an excitation source. ${ }^{5}$ Compared with the multiple phosphor approach, the single-phosphor approach eliminates the need to consider the mass ratio of several phosphors. Moreover, it has simplified the technology of device fabrication and reduced energy loss during the operation of the device. A typical yellow phosphor is $\mathrm{Y}_{3} \mathrm{Al}_{5} \mathrm{O}_{12}: \mathrm{Ce}^{3+}$, prepared with rare-earth materials. However, the high cost of raw materials and the environmental damage caused during the mining process are the fatal weaknesses limiting the widespread use of rare-earth phosphors. Furthermore, the relatively low color rendering index (CRI) of the white LEDs based on the $\mathrm{Y}_{3} \mathrm{Al}_{5} \mathrm{O}_{12}$ : $\mathrm{Ce}^{3+}$ yellow phosphor hinders the development of this phosphor for application in high-quality lighting and displays. ${ }^{6}$ Thus, new yellow fluorescent materials should be developed to replace the traditional phosphors.

Recently, a large number of studies have been conducted on the application of semiconductor quantum dots. ${ }^{7,8}$ However, the harsh preparation conditions and the unsolvable problem of toxicity still pose huge challenges to the industrial production as well as the use of semiconductor quantum dots. In fact, the low-cost and non-toxic fluorescent carbon materials are the optimal choices in this regard. Graphene quantum dots (GQDs), a new type of zero-dimensional carbon nanomaterials, have attracted significant attention since their first report in 2008. ${ }^{9}$ Owing to their low toxicity, excellent biological compatibility, facile synthesis and unique physical nature, GQDs can play an essential role in multiple fields (e.g., chemical sensing, ${ }^{\mathbf{1 0 , 1 1}}$ 
catalysis, ${ }^{12}$ and bioimaging $\left.{ }^{\mathbf{1 3 - 1 7}}\right)$. In addition to the abovementioned properties, excellent photoluminescence (PL) and high surface grafting make the GQDs useful for application in optoelectronic devices. ${ }^{\mathbf{1 8 - 2 8}}$ More importantly, as a novel phosphor, GQDs have the potential to substitute rare-earth phosphors in white LEDs. ${ }^{29}$

In most cases, there are two ways to apply the GQDs in LEDs. In general, luminescent materials directly serve as the emitting part. However, this requires accurate optimization of the device structure and technology. ${ }^{30}$ The other way is to use the lightemitting materials as the colour conversion layer of the lighting-emitting chip. ${ }^{31}$ The second way that deals with the colour conversion layer is relatively easier. Despite this, GQDs must be uniformly dispersed in a suitable solid matrix to avoid self-quenching caused by the aggregation of GQDs. In addition, their mechanical and chemical stabilities could be improved. ${ }^{32}$ In this study, yellow-green emitting GQDs served as the new phosphors. The as-prepared GQDs were cast in UV-curable siloxane, and a polymer film was formed with superior optical transparency and excellent monodisperse properties of GQDs. Via the simple adjustment of the thickness and the GQD concentration of the colour convert matrix, tunable colour temperatures (3196-10 $870 \mathrm{~K}$ ) of the GQD-based white LEDs were achieved, and their CIE coordinates matched well with the blackbody radiation curve. The polymeric matrix was applied in white LEDs with good luminescence quality and gratifying stability; this indicated the potential of the GQDs for application in high-end lighting.

\section{Experimental}

\section{Material and instruments}

We purchased the silicone resins ET-821A and ET-821B, a UV adhesive (U-613), blue-light-chips (EZ1000), and a silver adhesive (M705-S101-S4) from the market. Moreover, we used a spekron coating integrating sphere and a fluorescence spectrophotometer (HITACHI F4500) to investigate the electroluminescence properties and absorption characteristics. In addition, the PL properties were examined by the FLS920 fluorescence spectrometer. Transmission electron microscopy (TEM) was performed using the JEM-2100F electron microscope at the acceleration voltage of $200 \mathrm{kV}$ with a CCD camera. Fourier transform infrared (FTIR) spectra were obtained using the Nicolet AVATAR 360 FTIR instrument.

\section{Synthesis of GQDs}

Based on a previous study, ${ }^{33}$ the GQDs were synthesized by the following method. Typically, $0.05 \mathrm{~g}$ of 1,5-diaminonaphthalene was slowly added to a mixed solvent of $7 \mathrm{~mL}$ of ethanol and $3 \mathrm{~mL}$ of chloroform under stirring. The solution was sonicated for $30 \mathrm{~min}$. Then, the prepared solution was transferred to a $25 \mathrm{~mL}$ polytetrafluoroethylene autoclave and reacted at $180{ }^{\circ} \mathrm{C}$ for $12 \mathrm{~h}$. After being cooled down, the solution was taken out and filtered through a $220 \mathrm{~nm}$ filter. The resulting GQD solution was placed in a rotary evaporator; the organic solvent was evaporated to dryness $\left(50^{\circ} \mathrm{C}\right)$, and the GQD solid was added into other solvents, such as hexamethylene, toluene, ethylene glycol, ethanol, etc.

\section{Preparation of blue-light devices for experiment}

The blue-light chip was welded on the silver coating using a conductive silver paste, and a gold wire was bonded with the pad of the chip and the substrate. Subsequently, the silicone resins $\mathrm{A}$ and $\mathrm{B}$ were uniformly mixed (ET-821A : ET-821B = $4: 1$ ) using a centrifugal blender. To protect the chip from the solvent of GQDs, the silicone resin was covered on the LED chip. Then, the LED coated with the silicone resin mixture was heated for curing at $150{ }^{\circ} \mathrm{C}$ in a drying box for $10 \mathrm{~min}$.

\section{Fabrication of the white LEDs}

To attain a more stable external environment for the GQDs, they were introduced into a UV curable adhesive matrix in this study. As shown in Fig. 1, the approach used for preparing the GQD/UV

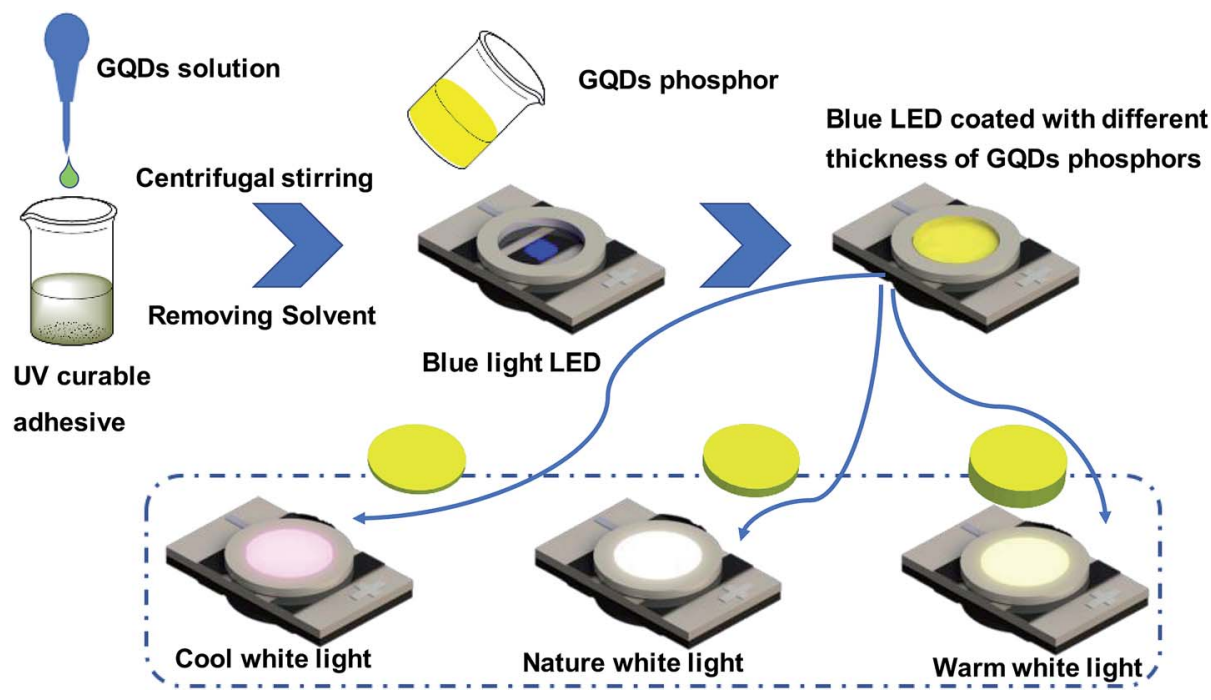

Fig. 1 Schematic of the fabrication of GQD-based tunable white LEDs. 
glue composite was straightforward. To realize a transparent GQD/UV glue mixture system, $200 \mu \mathrm{L}$ of GQD toluene solution at a concentration of $24 \mathrm{mg} \mathrm{mL} \mathrm{m}^{-1}$ and $2 \mathrm{~g}$ of UV glue were continuously centrifuged and stirred. To remove toluene from this dispersion, the composite system was repeatedly heated at $50{ }^{\circ} \mathrm{C}$ and centrifuged to eliminate the bubbles formed by heating. When no bubbles were generated, it meant that toluene was completely removed. Subsequently, the appropriate GQD/UV glue composite was deposited on the surface of the asprepared blue LEDs. Finally, the white LEDs were produced.

\section{Results and discussion}

The morphology of the GQDs was examined, as shown in Fig. 2. According to the TEM images (Fig. 2a and b), the as-prepared GQDs have a size distribution range from 1 to $7 \mathrm{~nm}$ with an average size of $4 \mathrm{~nm}$. The high-resolution TEM (HRTEM) image (Fig. 2c) presents the high crystallinity of the GQDs. The lattice spacing of $0.21 \mathrm{~nm}$ (Fig. 2c) was consistent with the in-plane lattice spacing of graphene (100). ${ }^{34,35}$ Furthermore, the peak located at $23.9^{\circ}$ corresponded to the (002) diffraction facet of graphite carbon (Fig. $\mathrm{S} 1 \dagger$ ). The uniform size distribution and clear lattice structure implied that the GQDs were successfully obtained. The AFM images of the GQDs (Fig. 2d) indicate that the average height of the GQDs is $3.46 \mathrm{~nm}$. Accordingly, this suggests that the GQDs are primarily multilayer structures. ${ }^{36}$

At first, UV-vis absorption and PL tests were carried out on the GQDs to investigate their PL properties. The PL test was performed by fixing the excitation wavelength and scanning the intensity of the light emitted from the toluene solution of the GQDs. Finally, the relationship between the emission wavelength and the wavelength of the incident light was obtained. As
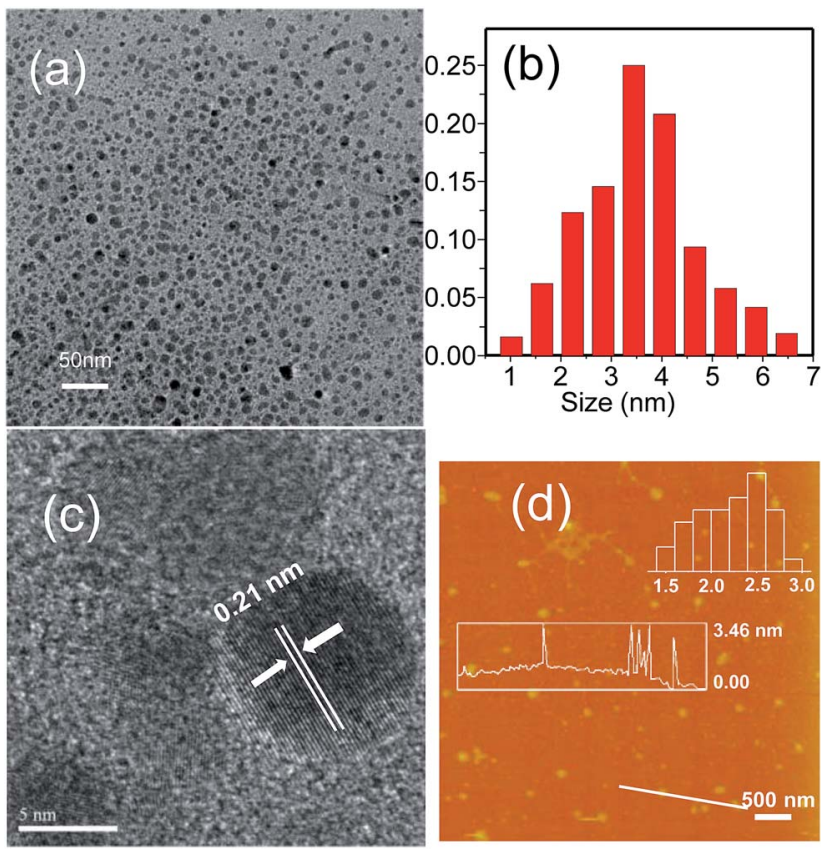

Fig. 2 (a) TEM image of the GQDs. (b) The distribution of particle size. (c) HRTEM image. (d) AFM image of the GQDs and their height distributions. shown in Fig. 3a, the emission wavelength was independent of the excitation wavelength. Moreover, the emission intensity of the GQD solution increased with a decrease in the excitation wavelength of the light. A slight red-shift (not more than $5 \mathrm{~nm}$ ) was found in the emission spectrum of the GQD toluene solution when the excitation wavelengths were $360 \mathrm{~nm}, 390 \mathrm{~nm}$, $420 \mathrm{~nm}$, and $450 \mathrm{~nm}$. Previous studies have suggested that the GQDs have excitation-independent property. ${ }^{37-39}$ In the UV-vis absorption spectra (Fig. 3b), the strong characteristic absorption peak at $324 \mathrm{~nm}$ corresponds to the $\mathrm{n} \rightarrow \pi^{*}$ transition of the $\mathrm{C} \equiv \mathrm{O}$ bond, ${ }^{40}$ and the relatively weak absorption peak at $407 \mathrm{~nm}$ is ascribed to doping with chlorine. ${ }^{41}$ Moreover, the excitation spectrum of the GQDs presents two peaks at $330 \mathrm{~nm}$ and $409 \mathrm{~nm}$ close to their UV-vis absorption peaks. For better comprehension, the PL spectra obtained using different solvents were compared. Fig. $3 c$ shows that the PL spectra of the GQDs dissolved in cyclohexane, toluene, ethylene glycol and ethanol solvents. Compared with the case of the toluene solution, as shown in Fig. 3c, the main peaks in the case of other solutions were slightly changed under excitation at $450 \mathrm{~nm} \cdot{ }^{42} \mathrm{In}$ addition to this, a splitting pattern was observed in the ethanol solution spectrum, and an anomaly peak located at $544 \mathrm{~nm}$ appeared. To investigate this, the functional groups of GQDs were identified by FTIR spectroscopy (Fig. S2 $\dagger$ ). The peak of C$\mathrm{Cl}$ at $535 \mathrm{~cm}^{-1}$ demonstrated the chlorine doping of GQDs. The double peak issue, as described in previous studies, arises from the different electronic transition pathways introduced into the band structure of GQDs as a result of chlorine doping. ${ }^{43}$ Moreover, no double-peak or multi-peak emission was observed in the group without chlorine doping. ${ }^{33}$ The abovementioned results suggested that the PL of the prepared GQDs originated not only from their quantum size effect but also from their surface functional groups.

GQDs at a concentration of $24 \mathrm{mg} \mathrm{mL}^{-1}$ were uniformly mixed with a UV curable glue at the mass ratios of $0.78 \mathrm{mg} \mathrm{g}^{-1}$, $0.1 \mathrm{mg} \mathrm{g}^{-1}, 0.01 \mathrm{mg} \mathrm{g}^{-1}$ and $0 \mathrm{mg} \mathrm{g}^{-1}$. Fig. 4a shows that the prepared GQD/UV adhesive composites change from colorless to buff to brown with the increasing GQD concentration. An image of the prepared GQD/UV adhesive composites with different concentrations of GQDs placed in ceramic cups under a UV lamp (wavelength: $365 \mathrm{~nm}$ ) is illustrated in Fig. 4b. Due to the PL properties of the prepared GQD/UV adhesive composites, when the UV lamp was turned on, the films turned brighter. With an increase in the concentration of GQDs in the gel, it was found that the emission peaks had a certain degree of redshift and became wider. The color of the emitted light was green inclined to yellow, as shown in Fig. 4b. The shift of the emission peaks and the wider PL spectra of the GQD/UV glue composites than those of the GQDs in solvents (Fig. 4c) originate from reabsorption. ${ }^{44}$ The red shift could be attributed to the increased absorption of the GQDs with a decrease in the excitation wavelength, as shown in the Fig. $3 \mathrm{~b}$. When the concentration of GQDs is high, the absorption degree of a short wavelength is much higher than that of a long wavelength. As a result, most of the short-wavelength light is absorbed and not emitted, and the long wavelengths easily penetrate the resin due to its lower absorption coefficient. ${ }^{45}$ 

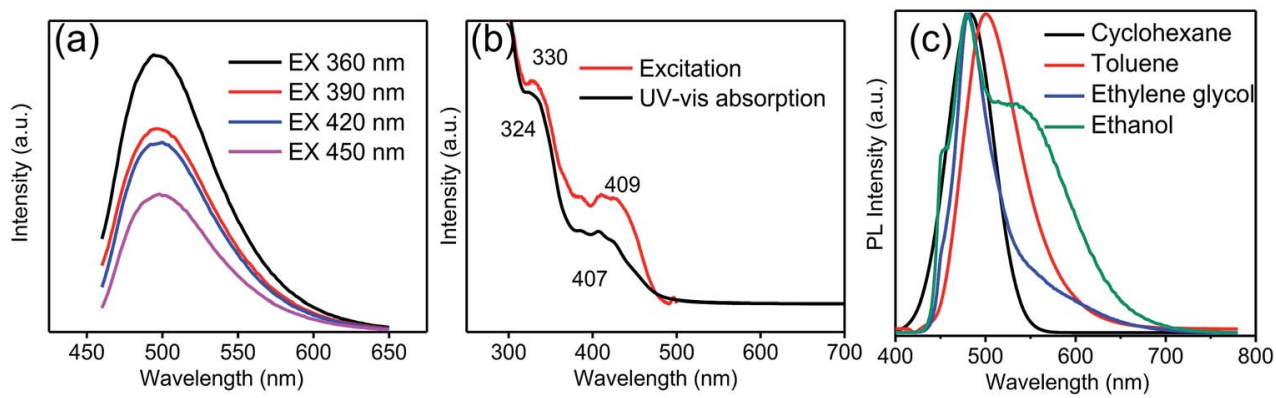

Fig. 3 (a) PL intensity of the GQDs in a toluene solution with various excitation wavelengths. (b) The UV-vis absorption (black line) and photoluminescence excitation (PLE) spectra (red line) of the GQDs in a toluene solution when the emission peak is located at $510 \mathrm{~nm}$. (c) Photoluminescence spectra of the GQDs in different solvents under $450 \mathrm{~nm}$ excitation.
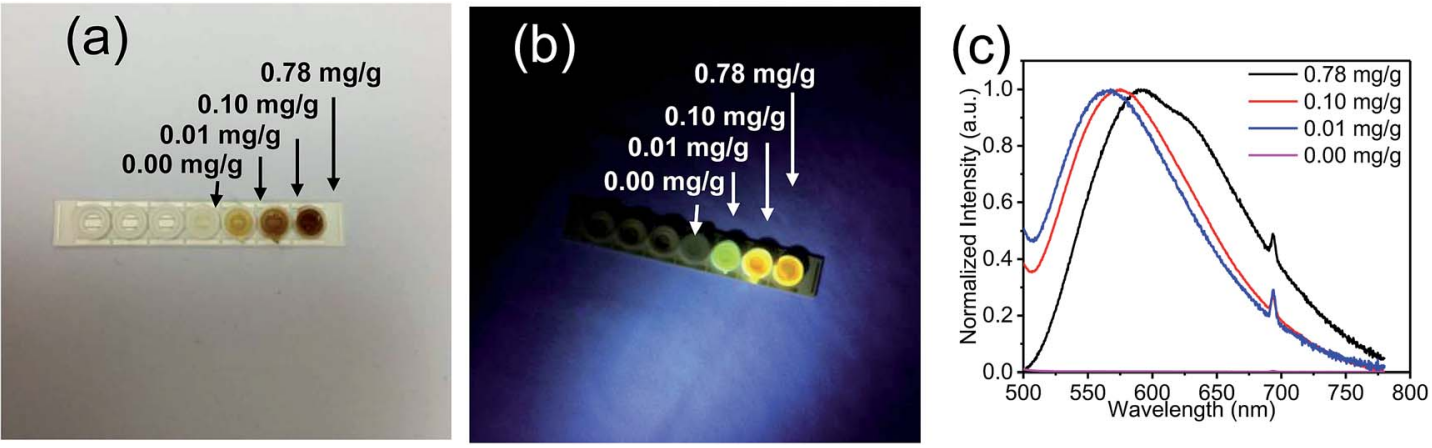

Fig. 4 Images of the films prepared with different concentrations of GQDs under (a) visible light and (b) UV light. (c) PL spectra of the films prepared with different concentrations of GQDs.

According to the aforementioned results, the GQDs in toluene were embedded in the gel with a strong fluorescence (Fig. 5). Taking Rhodamine B as a reference, we measured a quantum yield of $28 \%$ in the gel, slightly less than $31 \%$ in the solution ${ }^{33}$ this illustrated that it is suitable to embed the quantum dots in gels.

Furthermore, the mixture containing a suitable dose of GQDs was coated on the blue light-emitting InGaN diodes to fabricate white LEDs. These samples at three different concentrations showed the characteristic peak with a slight red shift. Fig. 5 presents the spectra of the LED samples under the three GQD concentration conditions. The coating thickness of the quantum dot gels on the LED chip was changed to realize the optimization of the spectra, great colour coordinates and colour temperature regulation. In this regard, the thick conversion layer worked as the optical path of the emitted light; the absorption of the emitted light enhanced with an increase in the optical path length. When the concentration was very low or very high, as shown in Fig. 5 a and c, the spectrum was slightly affected by the optical path (thickness of coating). In the case of a suitable concentration (Fig. 5b), the spectrum could be controlled well by the length of the optical path. As shown in Fig. 5 b, the blue light ratio decreased with an increase in the thickness of the colour conversion layer. Moreover, the correlated colour temperature decreased. The emission peak at $460 \mathrm{~nm}$ originated from the InGaN-based LEDs, and the broad
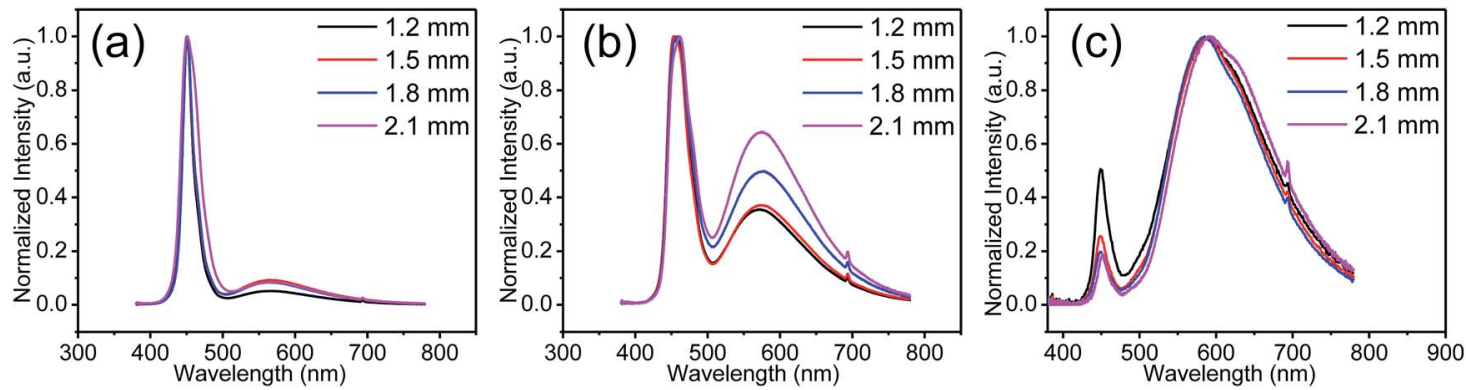

Fig. 5 The luminescence spectra of the LED samples coated with polymeric matrices of different thicknesses containing different GQDs concentrations: (a) $0.01 \mathrm{mg} \mathrm{g}^{-1}$, (b) $0.1 \mathrm{mg} \mathrm{g}^{-1}$, and (c) $0.78 \mathrm{mg} \mathrm{g}^{-1}$. 
emission peak near $580 \mathrm{~nm}$ covered almost the entire visible range from blue to red. This broader emission peak caused by the multiple reabsorptions of the initial emission peak results in a higher colour rendering index, which is crucial for improving the quality of light in LED illumination.

As shown in Fig. 6, the scattered dots in the CIE coordinates correspond to the LEDs with various concentrations and thicknesses of the coating. The inset images of the LEDs match with those obtained at the three concentrations. With an increase in the coating thickness, the dots moved from a low CCT position to a high CCT. Colour temperature-tunable white LEDs were achieved only by adjusting the concentration and thickness of the GQD films. Moreover, the CIE coordinates matched the blackbody radiation curve well. Table 1 shows that the higher the concentration of GQDs in the gel, the less the correlated colour temperature. This is because reabsorption will be greater with an increase in GQD content. ${ }^{46,47}$ White LEDs coated with low-concentration GQD/UV resin composites will have a higher luminous efficiency than those coated with high-concentration GQD/UV resin composites. The improvement of light efficiency is due to the increase in the

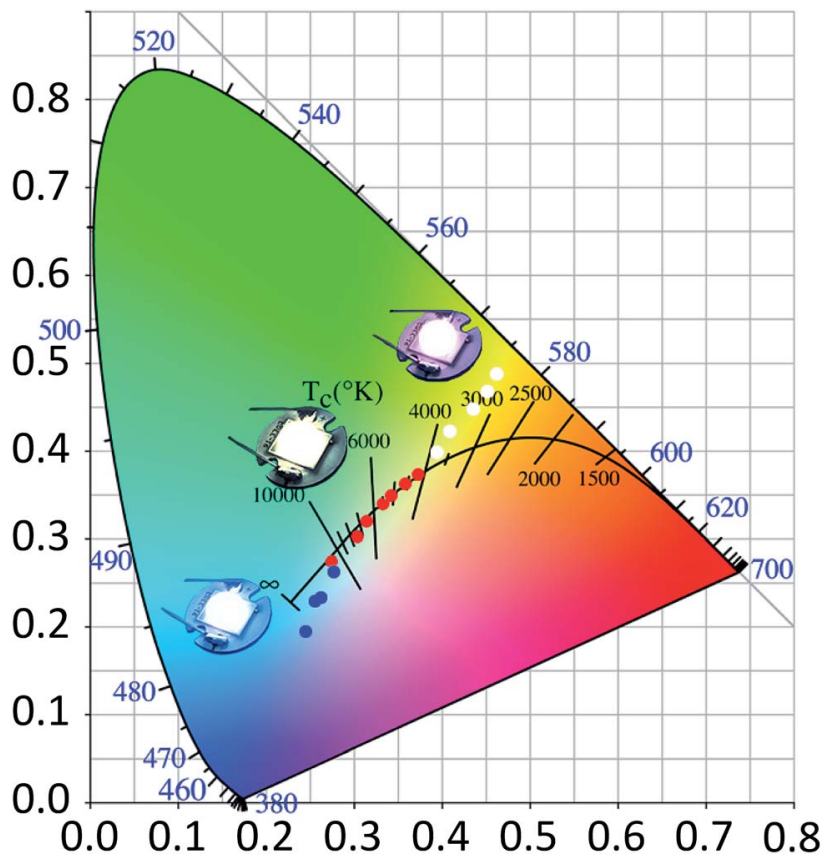

Fig. 6 Colour coordinates of the white LEDs lamps; inset: an image of white LED containing GQDs at various concentrations (blue dots represent low concentration condition; red dots represent medium concentration condition; white dots represent high concentration condition).

Table 1 CRI, CCT and luminous efficacy of LED samples with different GQDs (dissolved in toluene) concentrations

\begin{tabular}{llll}
\hline $\begin{array}{l}\text { Concentration } \\
\left(\mathrm{mg} \mathrm{g}^{-1}\right)\end{array}$ & CRI & $\begin{array}{l}\text { Luminous efficacy } \\
\left.(\operatorname{lm~W})^{-1}\right)\end{array}$ & CCT $(\mathrm{K})$ \\
\hline 0.01 & $32-61$ & $29-33$ & $>12862$ \\
0.10 & $83-86$ & $18-22$ & $4176-10870$ \\
0.78 & $66-73$ & $4-9$ & $3196-3802$
\end{tabular}
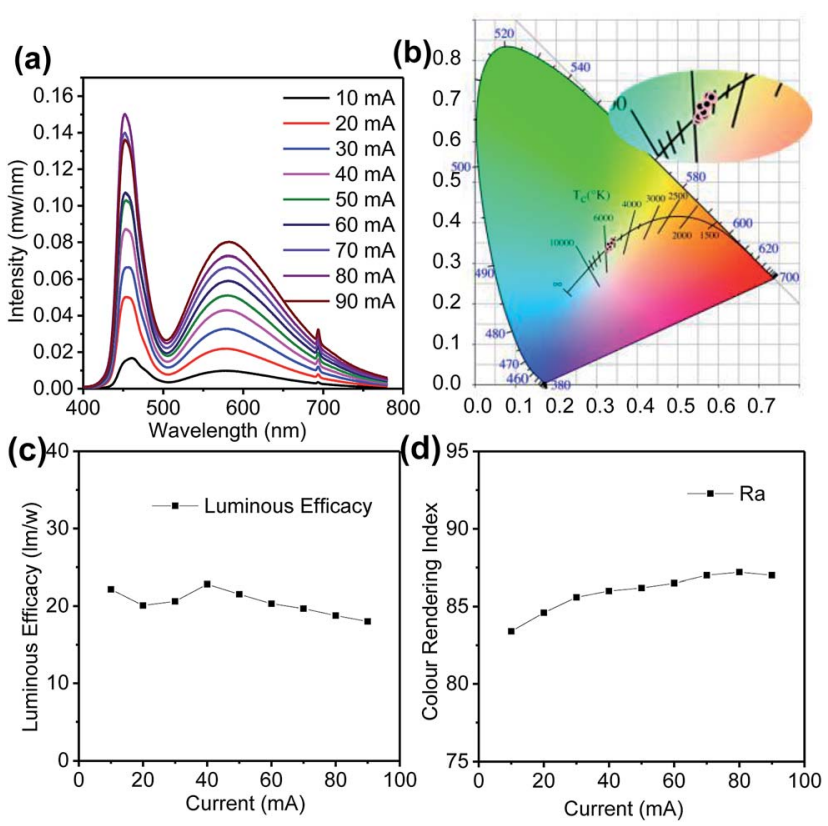

Fig. 7 (a) Electroluminescence spectrum, (b) CIE colour coordinates (c) luminous efficacy, and (d) colour rendering index of the GQDbased white LEDs under various working currents.

transparency caused by the decrease of GQD contents in the resin, and the light extraction becomes easy. When the mass of GQDs is $0.1 \mathrm{mg} \mathrm{g}^{-1}$, the sample has superior light quality. The device has a higher color rendering index than those fabricated with monochromatic phosphors. Moreover, the cost of the GQD synthesis is much lower than that of traditional quantum dots with the same level of luminous efficacy. ${ }^{48-51}$ GQDs in other solvents were also investigated, but their luminous efficiency was nearly one-third of that in toluene (Table S1 $\dagger$ ). The comparison of our results with those of other studies is presented in Table $\mathrm{S} 2 . \dagger^{52-58}$

To explore the characteristics of GQD-based white LED in depth, the device spectrum of colour conversion layer is measured under different working currents. Fig. 7a shows that with an increase in the working current, the emission intensity of the prepared device enhanced correspondingly. The spectrum of the GQD-based white LED (mass ratio UV resin : QDS = $1 \mathrm{~g}: 0.1 \mathrm{mg}$ ) presented peaks at $455 \mathrm{~nm}$ and $582 \mathrm{~nm}$, which did not shift with the increasing current. With an increase in current, the CIE chromaticity coordinates remained almost unchanged (Fig. 7b). According to Fig. 7c, the luminous efficacy changed slightly in the low working current range and dropped down when the current continued to increase, which is caused by the higher temperature under bigger operation current. Fig. $7 d$ displays that the colour rendering index varied from 84.4 to 87.2. In general, the device was maintained at a relatively stable status when loaded with different working currents. We also investigated the time stability of the device (Fig. S $3 \dagger$ ). The prepared device was kept in a drying cabinet environment for 6 months, and the luminous flux attenuation was less than $30 \%$, which indicated that the device had great stability. 


\section{Conclusions}

In conclusion, GQDs were successfully obtained from 1,5-diaminonaphthalene via a facile and cost-efficient route. The GQDs were introduced into a UV curable matrix and distributed uniformly without any surface treatment. This advantage is very significant for the application of GQDs in white LEDs. Moreover, we could realize spectrum tuning by only adjusting the concentration and thickness of the GQD films. Good quality white LEDs were obtained by coating a suitable GQD colour convert matrix with an appropriate GQD content on the blue light LEDs; furthermore, due to the use of the UV curable matrix, there is no need to consider the effect of high temperature caused by heat curing for silicone encapsulation; owing to the low cost and stability of the process, GQDs become a preferred candidate in the field of optoelectronic devices.

\section{Conflicts of interest}

There are no conflicts to declare.

\section{Acknowledgements}

This work was supported by the National Natural Science Foundation of China (No. 51605272, 21671129), the Shanghai Sailing Program (No. 16YF1404400), and the Shanghai Software And Integrated Circuit Industry Development special funds (170401). We thank the Laboratory for Microstructures of Shanghai University.

\section{References}

1 E. F. Schubert and J. K. Kim, Science, 2005, 308, 1274-1278.

2 Z. Liu, A. Ge, X. Tao, S. Yang and T. Wang, Opt. Spectrosc., 2016, 121, 72-77.

3 E. Jang, S. Jun, H. Jang, J. Lim, B. Kim and Y. Kim, Adv. Mater., 2010, 22, 3076.

4 X. Y. Lu, T. C. Hua, M. J. Liu and Y. X. Cheng, Thermochim. Acta, 2009, 493, 25-29.

5 S. Nakamura, S. Pearton and G. Fasol, The Blue Laser Diode, 2000.

6 N. C. George, K. A. Denault and R. Seshadri, Annu. Rev. Mater. Res., 2013, 43, 481-501.

7 X. Di, J. Jiang, Z. Hu, L. Zhou, P. Li, S. Liu, W. Xiang and X. Liang, Dyes Pigm., 2017, 146, 361-367.

8 J. Zhou, Z. Hu, L. Zhang and Y. Zhu, J. Alloys Compd., 2017, 708, 517-523.

9 L. A. Ponomarenko, F. Schedin, M. I. Katsnelson, R. Yang, E. W. Hill, K. S. Novoselov and A. K. Geim, Science, 2008, 320, 356-358.

10 A. Ananthanarayanan, X. Wang, P. Routh, B. Sana, S. Lim, D. H. Kim, K. H. Lim, J. Li and C. Peng, Adv. Funct. Mater., 2014, 24, 3021-3026.

11 P. G. Luo, S. Sahu, S. T. Yang, S. K. Sonkar, J. Wang, H. Wang, G. E. Lecroy, L. Cao and Y. P. Sun, J. Mater. Chem. B, 2013, 1, 2116-2127.

12 D. Pan, J. Jiao, Z. Li, Y. Guo, C. Feng, Y. Liu, L. Wang and M. Wu, ACS Sustainable Chem. Eng., 2015, 3, 2405-2413.
13 J. Peng, W. Gao, B. K. Gupta, Z. Liu, R. Romero-Aburto, L. Ge, L. Song, L. B. Alemany, X. Zhan, G. Gao, S. A. Vithayathil, B. A. Kaipparettu, A. A. Marti, T. Hayashi, J.-J. Zhu and P. M. Ajayan, Nano Lett., 2012, 12, 844-849.

14 L. Wang, W. Li, M. Li, Q. Su, Z. Li, D. Pan and M. Wu, ACS Sustainable Chem. Eng., 2018, 6, 4711-4716.

15 L. Wang, B. Wu, W. Li, Z. Li, J. Zhan, B. Geng, S. Wang, D. Pan and M. Wu, J. Mater. Chem. B, 2017, 5, 5355-5361.

16 L. Wang, W. Li, B. Wu, Z. Li, D. Pan and M. Wu, Chem. Eng. J., 2017, 309, 374-380.

17 L. Wang, W. Li, B. Wu, Z. Li, S. Wang, Y. Liu, D. Pan and M. Wu, Chem. Eng. J., 2016, 300, 75-82.

18 X. Guo, C. F. Wang, Z. Y. Yu, L. Chen and S. Chen, Chem. Commun., 2012, 48, 2692-2694.

19 Y. P. Sun, B. Zhou, Y. Lin, W. Wang, K. A. Fernando, P. Pathak, M. J. Meziani, B. A. Harruff, X. Wang and H. Wang, J. Am. Chem. Soc., 2006, 128, 7756-7757.

20 S. Y. Lim, W. Shen and Z. Gao, Chem. Soc. Rev., 2015, 44, 362. 21 Q. L. Chen, C. F. Wang and S. Chen, J. Mater. Sci., 2013, 48, 2352-2357.

22 F. Bonaccorso, Z. Sun, T. Hasan and A. C. Ferrari, Nat. Photonics, 2010, 4, 611-622.

23 J. Shen, Y. Zhu, X. Yang and C. Li, Chem. Commun., 2012, 43, 3686-3699.

24 X. Miao, S. Tongay, M. K. Petterson, K. Berke, A. G. Rinzler, B. R. Appleton and A. F. Hebard, Nano Lett., 2012, 12, 2745.

25 S. Paulo, E. Palomares and E. Martinezferrero, Nanomaterials, 2016, 6, 157.

26 W. Li, M. Li, Y. Liu, D. Pan, Z. Li, L. Wang and M. Wu, ACS Appl. Nano Mater., 2018, 1, 1623-1630.

27 S. Diao, X. Zhang, Z. Shao, K. Ding, J. Jie and X. Zhang, Nano Energy, 2017, 31, 359-366.

28 C. Sun, Y. Zhang, K. Sun, C. Reckmeier, T. Zhang, X. Zhang, J. Zhao, C. Wu, W. W. Yu and A. L. Rogach, Nanoscale, 2015, 7, 12045-12050.

29 L. H. Mao, W. Q. Tang, Z. Y. Deng, S. S. Liu, C. F. Wang and S. Chen, Ind. Eng. Chem. Res., 2014, 53, 6417-6425.

30 F. Wang, Y. H. Chen, C. Y. Liu and D. G. Ma, Chem. Commun., 2011, 47, 3502-3504.

31 X. Zheng, H. Wang, Q. Gong, L. Zhang, G. Cui, Q. Li, L. Chen, F. Wu and S. Wang, Mater. Lett., 2015, 143, 290-293.

32 X. Li, M. Rui, J. Song, Z. Shen and H. Zeng, Adv. Funct. Mater., 2015, 25, 4929-4947.

33 L. Wang, B. Wu, W. Li, S. Wang, Z. Li, M. Li, D. Pan and M. Wu, Adv. Biosyst., 2018, 2, 1700191.

34 Y. Dong, H. Pang, H. B. Yang, C. Guo, J. Shao, Y. Chi, C. M. Li and T. Yu, Angew. Chem., Int. Ed. Engl., 2013, 125, 7954-7958.

35 L. Wang, Y. Wang, T. Xu, H. Liao, C. Yao, Y. Liu, Z. Li, Z. Chen, D. Pan, L. Sun and M. Wu, Nat. Commun., 2014, $5,5357$.

36 S. Zhu, J. Zhang, C. Qiao, S. Tang, Y. Li, W. Yuan, B. Li, L. Tian, F. Liu and R. Hu, Chem. Commun., 2011, 47, 6858-6860.

37 T.-F. Yeh, W.-L. Huang, C.-J. Chung, I. T. Chiang, L.-C. Chen, H.-Y. Chang, W.-C. Su, C. Cheng, S.-J. Chen and H. Teng, J. Phys. Chem. Lett., 2016, 7, 2087-2092.

38 Q. Xue, H. Huang, L. Wang, Z. Chen, M. Wu, Z. Li and D. Pan, Nanoscale, 2013, 5, 12098-12103. 
39 Y. Dong, H. Pang, H. B. Yang, C. Guo, J. Shao, Y. Chi, C. M. Li and T. Yu, Angew. Chem., Int. Ed., 2013, 52, 7800-7804.

40 F. Zhang, X. Feng, Y. Zhang, L. Yan, Y. Yang and X. Liu, Nanoscale, 2016, 8, 8618-8632.

41 D. Qu, M. Zheng, P. Du, Y. Zhou, L. Zhang, D. Li, H. Tan, Z. Zhao, Z. Xie and Z. Sun, Nanoscale, 2013, 5, 12272-12277.

42 H. Wang, C. Sun, X. Chen, Y. Zhang, V. L. Colvin, Q. Rice, J. Seo, S. Feng, S. Wang and W. W. Yu, Nanoscale, 2017, 9, 1909-1915.

43 X. Li, S. P. Lau, L. Tang, R. Ji and P. Yang, J. Mater. Chem. C, 2013, 1, 7308-7313.

44 S. Javanbakht and H. Namazi, Carbohydr. Polym., 2017, 176, 220-226.

45 Y. Wang, S. Kalytchuk, Y. Zhang, H. Shi, S. V. Kershaw and A. L. Rogach, J. Phys. Chem. Lett., 2014, 5, 1412-1420.

46 Y. Zhai, Z. Ding, P. Jing, L. Di, H. Zeng and S. Qu, J. Colloid Interface Sci., 2017, 497, 165-171.

47 C. Sun, Y. Zhang, S. Kalytchuk, Y. Wang, X. Zhang, W. Gao, J. Zhao, K. Cepe, R. Zboril, W. W. Yu and A. L. Rogach, J. Mater. Chem. C, 2015, 3, 6613-6615.

48 H. S. Jang, H. Yang, S. W. Kim, J. Y. Han, S.-G. Lee and D. Y. Jeon, Adv. Mater., 2008, 20, 2696-2702.
49 A. Aboulaich, M. Michalska, R. Schneider, A. Potdevin, J. Deschamps, R. Deloncle, G. Chadeyron and R. Mahiou, ACS Appl. Mater. Interfaces, 2014, 6, 252-258.

50 P. H. Chuang, C. C. Lin and R. S. Liu, ACS Appl. Mater. Interfaces, 2014, 6, 15379-15387.

51 I. S. Sohn, S. Unithrattil and W. B. Im, ACS Appl. Mater. Interfaces, 2014, 6, 5744-5748.

52 C. M. Luk, L. B. Tang, W. F. Zhang, S. F. Yu, K. S. Teng and S. P. Lau, J. Mater. Chem., 2012, 22, 22378-22381.

53 S. Lin, M. Chen, Z. Wang, Y. Zhang, R. Yuan, X. Liang, W. Xiang and Y. Zhou, Chem. Eng. J., 2017, 324, 194-202.

54 X. F. Wang, G. G. Wang, J. B. Li, Z. Liu, W. F. Zhao and J. C. Han, Chem. Eng. J., 2018, 336, 406-415.

55 T. Zhang, F. Zhao, L. Li, B. Qi, D. Zhu, J. Lü and C. Lü, ACS Appl. Mater. Interfaces, 2018, 10, 19796-19805.

56 J. Zhu, X. Bai, J. Bai, G. Pan, Y. Zhu, Y. Zhai, H. Shao, X. Chen, B. Dong, H. Zhang and H. Song, Nanotechnology, 2018, 29, 085705.

57 L. Li and T. Dong, J. Mater. Chem. C, 2019, 7, 3105.

58 X. F. Wang, G. G. Wang, J. B. Li, Z. Liu, Y. X. Chen, L. F. Liu and J. C. Han, Chem. Eng. J., 2019, 361, 773-782. 